New techniques in medicine

\title{
Transoesophageal echocardiography
}

\author{
John E Sanderson, Wilson WM Chan
}

\begin{abstract}
Summary
Transoesophageal echocardiography gives unparalleled views of the posterior structures of the heart. It is the investigation of choice for the diagnosis of acute dissection of the aorta, in patients with endocarditis, management of the hypotensive patient in the intensive care unit, and in the search for a potential cardiac source of thromboembolism. However, it is a semi-invasive procedure, and proper training is required to carry it out safely, to ensure that maximum information is obtained at the time of examination, and to avoid diagnostic pitfalls.
\end{abstract}

Keywords: transoesophageal echocardiography

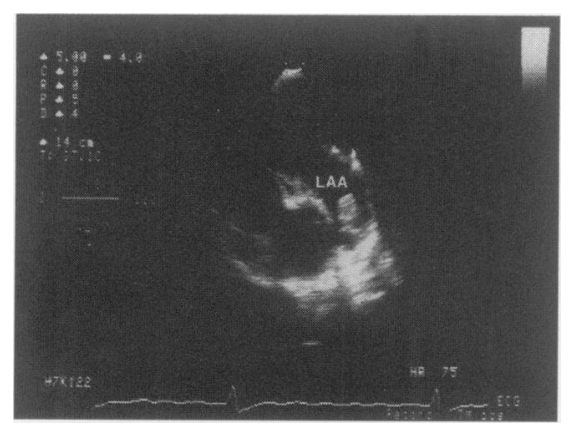

Figure 1 Thrombus present in the left atrial appendage (LAA)
Transthoracic echocardiography has become one of the most useful techniques in cardiology. However, it is not always possible to get good views via the chest, especially of the posterior structures, the mitral valve and left atrium. The idea of looking at the heart from the oesophagus is not new and in 1976, Frazin et al described their initial experience with an ultrasound transducer that was passed into the oesophagus but the transducer was unstable. ${ }^{1}$ In the 1980 s the ultrasound transducers were attached to flexible endoscopes, giving easier positioning of the probes and the development of transoesophageal echocardiography (TOE). Further advances have occurred and now probes are smaller and capable of multiplane viewing by mechanically or electronically rotating the scanning plane through $180^{\circ}$.

\section{The transoesophageal echocardiographic examination}

Like upper gastrointestinal endoscopy, TOE is an invasive procedure and should only be performed by those who have received adequate training. The advantage of TOE is that it can be performed virtually anywhere, in wards, outpatients, the operating room or in Accident and Emergency departments. The patient should fast for at least four hours before and it is important to exclude any history of dysphagia or gastro-oesophageal disease. In most patients the study can be done without any premedication. Patients with suspected aorta dissection or those who are uncooperative, may need sedation. Usually antibiotic prophylaxis is not necessary. A pulse oximeter probe and automated blood pressure device are usually recommended. The risk associated with TOE is low. In the European multicentre study, ${ }^{2}$ out of 10419 TOE examinations, the probe could not be inserted successfully in only $1.9 \%$, and the examination had to be interrupted because of the patient's inability to tolerate the probe, primary cardiac or bleeding related complications in $0.9 \%$. One patient died during the procedure (mortality $0.01 \%$ ).

Although the aim of the study should be to answer the main clinical question poised, it is important to do a complete examination of all regions of the heart and the great vessels. The usual scanning planes, horizontal and vertical, are illustrated in figure 1.

\section{Pitfalls of TOE}

Because endocardial structures are seen so clearly with TOE, some normal variations may be misdiagnosed as pathological. The most common pitfalls are listed below:

- Atrium muscular trabeculations and irregularities are seen in the walls of both atrial appendages. These muscle ridges are usually small, reflectile and typically multiple. They have to be distinguished from thrombus which is characteristically of a different texture than the atrial wall and more echo refractile with uniform consistency. Tumours which extend into the atria also have a different echo density. On occasion, the origin of the pulmonary veins are encircled by tissue that may appear mass-like in some sections. The wall separating the left atrial appendage from left upper pulmonary vein in certain sections can look particularly globular and therefore like a mass mimicking a left atrial tumour. In the right atrium the orifice of the superior and inferior vena cava often has muscle bundles that can appear mass-like.

- Atrial septum. Occasionally fat-laden masses are found in the septum around the fossa ovalis. In older patients the fat in this area can be up to $1 \mathrm{~cm}$ thick and occasionally larger.

- Tricuspid annulus. Fatty tissue is often present at the free wall at the end of the tricuspid annulus. Occasionally this is quite large and can produce a mass-like effect. It is important not to misinterpret this as a tumour or a ring abscess.

- Aortic valve. If the aortic valve leaflets are cut obliquely, then the normal valve appears to have a mass which may be incorrectly thought to be a 


\begin{tabular}{|l|}
\hline Common indications for \\
TOE \\
\hline - cardiac source of embolism \\
- endocarditis \\
- dissection of the aorta \\
assessment of prosthetic valve \\
- assessment of native valve function \\
- cardiac neoplasms \\
- in critically ill (hypotensive) patients \\
operative \\
\hline
\end{tabular}

Box 1

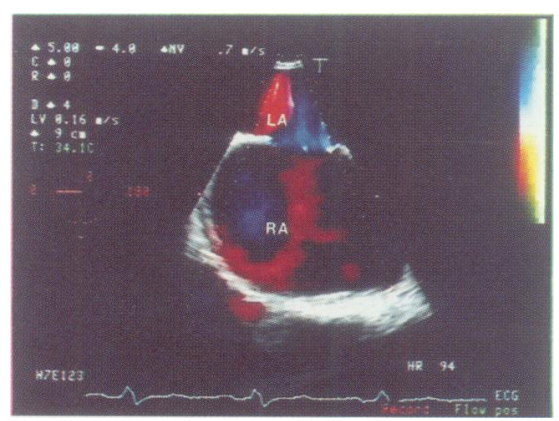

Figure 2 Secundum atrial septal defect between left atrium (LA) and right atrium (RA). In this particular example the RA is much enlarged with flow demonstrated by colour-flow Doppler passing from the RA to the LA, ie, reversed shunting due to Eisenmenger's syndrome

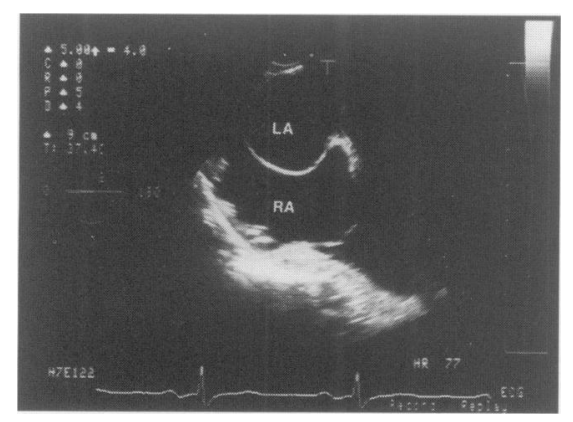

Figure 3 Example of an atrial septal aneurysm with bulging of the septum towards the right atrium (RA); LA = left atrium vegetation. Multiplane views of the aortic valve eliminate this potential pitfall.

- Membranes. At the orifice of the inferior vena cava, in the right atrium the eustachian valve often appears as a mobile, undulating membrane, which can be mistaken for a thrombus. Multiplane imaging allows accurate identification. Similarly, a thin membrane or a forme fruste of cor triatum appears as an incomplete membrane in the left atrial cavity. Usually this arises from the posterior margin of the membrane of the fossa ovalis and inserts into the common wall separating the left upper pulmonary vein and left atrial appendage. Sometimes the overlap between a fatty atrial septum and the fossa membrane in certain planes can appear as a cavity.

- Echo-free spaces. The transverse sinus is a pericardial reflection between the left atrium and the great vessels at the base of the heart typically contains a small amount of pericardial fluid. This produces a small echo-free space between the left atrium and the aorta in the horizontal plane and a triangular space in the longitudinal plane. Occasionally, epicardial fat within it can appear as a mobile cyst or solid mass. Similarly, the oblique sinus is a fluidfilled space interposed between the left atrium and the oesophagus.

For further details and examples of these common pitfalls see Seward et al. ${ }^{3}$

\section{Clinical indications for TOE}

The main indications for TOE are shown in box 1 .

\section{SOURCE OF EMBOLISM}

In clinical studies, cardiogenic embolism has been shown to account for approximately $15 \%$ (6-23\%) of ischaemic strokes. ${ }^{4,5}$ 'Lone' or nonvalvular atrial fibrillation is the underlying cause in about $45 \%$ and the remainder is equally divided among patients with acute myocardial infarction, left ventricular aneurysm, rheumatic heart disease, prostatic valve and a variety of other causes. ${ }^{4,5}$ Since TOE can detect thrombus much more readily than thoracic echocardiography especially in the left atrium (figure 1) it has been widely used for screening patients for cardiogenic embolism. However, a distinction must be made between detecting a direct source of embolism, such as the presence of an obvious atrial thrombus, as opposed to an indirect (or possible) source such as mitral valve prolapse or an atrial septum aneurysm. Demonstrating the presence of such lesions is not proof that they were the origin of the embolic thrombus.

In patients who clinically have a low suspicion for cardiogenic embolism, the detection of a direct source of embolism is very low, even by TOE. There is debate about how frequently TOE should be done in patients with strokes and there are no adequate guidelines at present. In general TOE should be done when there is a strong clinical suspicion of an embolic cause of stroke (and transthoracic echocardiography is not helpful) in younger patients. However it is clear that TOE is better than transthoracic echocardiography for detection of left atrial thrombus and other potential causes of thrombo-embolism. In a metaanalysis of studies that included 1469 patients who had cerebral ischaemia, peripheral arterial embolism, non valvular atrial fibrillation or who were candidates for mitral valvuloplasty, 183 patients $(12.5 \%)$ had thrombi in the left atrial appendage detected by TOE, ${ }^{6}$ but thrombi were detected by transthoracic echocardiography in only two patients. In addition to detecting thrombus in the left atrial appendage, TOE also provides high quality images of the atrial septum. Patent foramen ovale, or small secundum atrial septal defects which allow paradoxical emboli can be identified easily, especially with colourDoppler or saline contrast (figure 2). In addition, atrial septal aneurysms (figure 3) are increasingly recognised and do appear to be associated with an increased risk of embolism. ${ }^{7}$

Spontaneous echo contrast, which indicates slow blood flow is also seen more frequently by TOE. ${ }^{8}$ It is found commonly in patients with mitral valve stenosis, mitral valve replacements or atrial fibrillation. In patients with either mitral stenosis or mitral prosthesis, Black et al found that spontaneous contrast is the only independent predictor of thrombus formation, a history of suspected embolism, or both. 9 They also indicate an increased risk of thrombus formation and embolism in patients with non-valvular atrial fibrillation. ${ }^{9}$ Vegetations (see below) may also be a source of embolism and are more clearly seen by TOE. Recently, there has been increasing interest in the detection of aortic atheroma by TOE. In a study by Tunick et al, atheroma seen in the aortic arch was found in 33 of 122 patients with a history of stroke, transient ischaemic attack or peripheral embolism but in only 11 of 122 controls without history of these events. ${ }^{10}$ However, the correct management of these patients (to anticoagulate or not) is still unclear. 


\begin{tabular}{|l|}
\hline Indications for TOE in \\
endocarditis \\
\hline - blood cultures 'negative' with high \\
clinical suspicion \\
- prolonged clinical course \\
- persistent fever or raised CRP \\
- deteriorating haemodynamics/ \\
increasing valvular regurgitation \\
- prosthetic valve endocarditis \\
\hline
\end{tabular}

Box 2

\section{ENDOCARDITIS}

The diagnosis of infective endocarditis still depends on the combination of clinical signs and blood cultures. However, the demonstration of vegetations can support the diagnosis or, in cases where the blood culture is negative, indicate a search for unusual organisms. TOE is substantially better than transthoracic echocardiography for the detection of vegetations. In one study of patients with endocarditis confirmed by surgery or at autopsy, vegetations were detected by transthoracic echocardiography in $58 \%$ and by TOE in $90 \% .^{11}$ Shively et al reported that TOE had a sensitivity and a specificity of 94 and $100 \%$, respectively, for the detection of vegetations. ${ }^{12}$ TOE cannot rule out the presence of endocarditis completely but the probability of the disease is very low if a TOE examination is completely normal. Moreover, the size of vegetations is important and some studies have indicated that vegetations $>10 \mathrm{~mm}$ in diameter have a greater risk of embolism. ${ }^{11}$ TOE is also superior to transthoracic echocardiography for the detection of abscesses. In the study by Daniel et al the sensitivity and specificity for the detection of abscesses was 87 and $95 \%$ for TOE and 28 and $99 \%$ for transthoracic echocardiography. ${ }^{13}$ TOE therefore is indicated in patients where there is a clinical suspicion of endocarditis and a transthoracic echocardiogram or blood cultures are inconclusive, in all patients with a prolonged clinical course, persistent signs of infection or lack of response to antibiotic therapy, deteriorating haemodynamics or increasing regurgitation, and in patients with prosthetic valve endocarditis.

\section{AORTIC DISSECTION}

TOE is now the diagnostic tool of choice. ${ }^{14,15}$ Nienaber et al ${ }^{16}$ compared computed tomography (CT), magnetic resonance imaging (MRI) and TOE, the sensitivities for detecting proximal aortic dissection were similar at 98.3, 97.7, and $93.8 \%$, respectively. In this particular study, the specificity of TOE $(77 \%)$ was lower than CT $(87.1 \%)$ or MRI $(97.8 \%)$ mainly as a result of false-positive findings in the ascending aorta. MRI and TOE were better than CT for identifying the entry side or aortic regurgitation. However, the results with multiplane TOE are likely to be better and in other studies TOE has been shown to have a sensitivity and specificity of 99 and $98 \%$, respectively, for diagnosis of aortic dissection, compared with 88 and $94 \%$, respectively, for angiography (table). ${ }^{17}$ TOE has the advantage of being portable and usually readily available, so that these patients can be studied in the intensive care unit, if necessary. The hallmark of a dissection on a TOE is the intimal flap (figure 4). Occasionally in the longitudinal view it can be seen to be undulating and there may be systolic expansion of the true lumen and compression of the false lumen. The false lumen may contain contrast echos due to the low velocity flow and variable amounts of thrombus are seen. In addition, with TOE, the degree of aortic regurgitation (in Type A dissection), presence of pericardial effusion, left ventricular function and involvement of the coronary arteries by the dissection can be assessed.

\section{PROSTHETIC HEART VALVES}

Studies have demonstrated that TOE is superior to transthoracic echocardiography for the assessment of prosthetic valves and provides more anatomical and functional information. ${ }^{18-20}$ Degeneration of the leaflets of biological valves, attachment of thrombi or vegetations can be seen more clearly and the differences between transvalvular and paravalvular leaks can be more reliably differentiated.

\section{TOE IN THE INTENSIVE CARE UNIT}

The use of TOE in critically ill patients in the intensive care unit is increasing, especially in patients with unexplained hypotension. In a study by Foster and

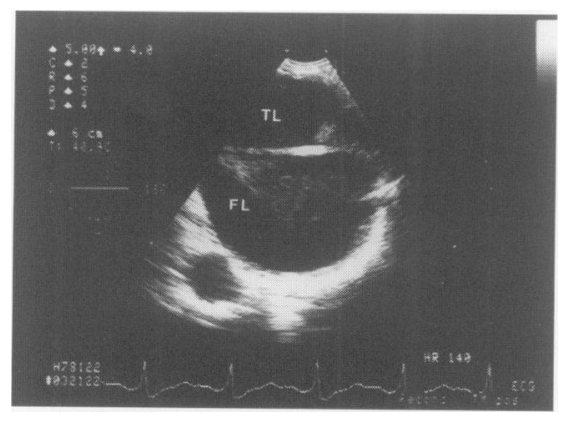

Figure 4 Examples of dissection of the aorta showing the true lumen (TL) and large false lumen (FL) divided by the intimal flap
Table TOE and suspected dissection of the aorta (from ref 17)

\begin{tabular}{llll}
\hline & TOE & $C T$ & Aortography \\
\hline Sensitivity & +++ & ++ & ++ \\
Specificity & $++l+++$ & +++ & +++ \\
Site of intimal tear & ++ & + & ++ \\
Presence of thrombus & + & ++ & +++ \\
Presence of aortic regurgitation & +++ & - & +++ \\
Pericardial effusion & +++ & ++ & + \\
Branch vessel involvement & + & + & ++ \\
Coronary artery involvement & + & - & ++ \\
\hline
\end{tabular}

$+++=$ excellent; $++=$ good; $+=$ fair and $-=$ not detected. 
Schiller of TOE in 69 critically ill patients, unexpected findings were identified in $25 \%$ which led to treatment changes in $17 \%$ of the patients. ${ }^{21}$ Similarly Oh et $a l^{22}$ found that TOE demonstrated the cause of illness in $59 \%$ of 51 patients in the intensive care unit who had inadequate transthoracic echocardiograms and in $24 \%$ of these, the findings of TOE led to cardiac surgery.

\section{TOE DURING SURGERY}

Interoperative TOE with colour-Doppler has replaced traditional procedures for the assessment of the adequacy of mitral and tricuspid valve repair. ${ }^{23}$ In addition, TOE is increasingly used for assessment of left ventricular function and the early detection of myocardial ischaemia during and immediately after cardiac or noncardiac surgery. ${ }^{24,25}$

\section{CONGENITAL HEART DISEASE}

With the availability of smaller probes, TOE is increasingly used in children and adults with congenital heart disease. In children the use of TOE must be balanced against the requirement for heavy sedation and the fact that the current probes are not ideal. In particular, cyanotic patients or those with low cardiac output should be monitored very carefully during the TOE procedure.

\section{OTHER CONDITIONS}

TOE is very useful for detecting and assessing atrial tumours, including tumours spreading into the pulmonary veins. Another area which is of research interest is the detection of coronary artery blood flow. Proximal segments of the coronary arteries can be visualised by colour flow Doppler with TOE and with pulsed Doppler coronary blood flow and coronary flow reserve estimated. ${ }^{26}$

\section{Conclusion}

TOE is an extremely useful tool for the assessment of cardiac patients. The main indications for its use are the diagnosis of acute dissection of the aorta, assessment of patients with endocarditis and searching for a potential cardiac source of embolism and for these particular conditions TOE is now the investigation of choice.

1 Frazin L, Talano JV, Stephanides L, Loeb HS, Kopel L, Gunnar RM. Esophageal echocardiography. Circulation 1976; 54: $102-8$.

2 Daniel WG, Erbel R, Kasper W, et al. Safety of transesophageal echocardiography: a multicente survey of 10,419 examinations. Circulation 1991 ; 83: $817-21$.

3 Seward JB, Khandheria BK, Oh JK, et al. Critical appraisal of transoesophageal echocardiography, limitations, pitfalls and complications. $\mathcal{F} \mathrm{Am}$ Soc Echocardiogr 1992; 5: 288-305.
litations, pitfalls and complication

4 Cerebral Embolism Task Force. Cardiogenic brain embolism. Arch Neurol 1986: 43: 71-84.

5 brain embolism. Arch Neurol 1986: 43: 71-84. brain embolism. The second report of the Cerebral Embolism Task Force. Arch Neurol 1989; 46: 727-43.

6 Mugge A, Kuhn H, Daniel WG. The role of transesophageal echocardiography in the detection of left atrial thrombi. Echocardiography 1993; 10: 405-17.

7 Schneider B, Hanrath P, Vogel P, Meinertz T. Improved morphologic characterization of atrial septal aneurysm by transesophageal echocardiography: relation to cerebrovascular events. $\mathcal{F} \mathrm{Am}$ Graphy: relation to cerebrovascular

8 Merino A, Hauptmann P, Badimon L, et al. Merino A, Hauptmann P, Badimon $\mathrm{L}$, et al. interaction of erythrocytes and plasma proteins modulated by shear forces. Am Coll Cardiol 1992; 20: 1661 - 8 .

9 Black IW, Hopkins AP, Lee LCL, Walsh WF. Left atrial spontaneous echo contrast: a clinical and echocardiographic analysis. $f \mathrm{Am}$ Coll Cardiol 1991; 18: 398-404.

10 Tunick PA, Perez JL, Kronzon I. Protruding atheromas in the thoracic aorta and systemic embolization. Ann Intern Med 1991; 115: 423-7.
11 Mugge A, Daniel WG, Frank G, Lichtlen PR. Echocardiography in infective endocarditis: reassessment of the prognostic implications vegetation size determined by the transthoracic and transesophageal approach. $f \mathrm{Am}$ Coll Cardiol 1989; 14: 631-8.

12 Shively BK, Gurule FT, Roldan CA, Leggett JH, Schiller NB. Diagnostic value of transesophageal compared with transthoracic echocardiography in infective endocarditis. $₹ \mathrm{Am}$ Coll Cardiol 1991; in infective $391-7$.

13 Daniel WG, Mugge A, Martin RP, et al. Improvement in the diagnosis of abscesses associated with endocarditis by transesophageal echocardiography. N Engl f Med 1991; 324: 795-800.

14 Cigarroa JE, Isselbacher EM, DeSanctis RW, Eagle KA. Diagnostic imaging in the evaluation of suspected aortic dissection: old standards and new directions. $N$ Engl $f$ Med 1993; 328: 35-43.

15 Erbel R, Engberding R, Daniel W, Roelandt J, Visser C, Rennollet H. Echocardiography in diagnosis of aortic dissection. Lancet 1989; 1: diagnosis

16 Nienaber CA, von Kodolitsch Y, Nicolas V, et al. The diagnosis of thoracic aortic dissection by
at et noninvasive imaging procedures. $N \mathrm{Engl} f \mathrm{Med}$ 1993; 328: $1-9$.

17 Cigarroa JE, Isselbacher EM, DeSanctis RW, Eagle KA. Diagnostic imaging in the evaluation of suspected aortic dissection: old standards and new directions. $N$ Engl $\mathcal{f}$ Med 1993; 328: 35-43.

18 Khandheria BK, Seward JB, Oh JK, et al. Value and limitations of transesophageal echocardiography in assessment of mitral valve prostheses. Circulation 1991; 83: 1956-68.
19 van den Brink RBA, Visser CA, Basart DCG, Duren DR, de Jong AP, Dunning AJ. Comparison of transthoracic and transesophageal color Doppler flow imaging in patients with mechanical prostheses in the mitral valve position. $A m \mathcal{f}$ Cardiol 1989; 63: 1471-4.

$20 \mathrm{Karalis}$ DG, Chandrasekaran K, Ross JJ Jr, et al. Single-plane transesophageal echocardiography for assessing function of mechanical or bioprosthetic valves in the aortic valve position. Am f thetic valves in the aortic valy
Cardiol 1992; 69: 1310-5.

21 Foster E, Schiller NB. The role of transesophageal echocardiography in critical care: UCSF experience. $₹$ Am Soc Echocardiogr 1992; 5: 368-74.

$22 \mathrm{Oh} \mathrm{JK}$, Seward JB, Khandheria BK, et al. Transesophageal echocardiography in critically ill patients. Am $₹$ Cardiol 1990; 66: 1492-5.

23 Maurer G, Siegel RJ, Czer LSC. The use of color flow mapping for intraoperative assessment of valve repair. Circulation 1991; 84 (suppl): I $250-8$.

24 Eisenberg MJ, London MJ, Leung JM, et al. Monitoring of myocardial ischemia during noncardiac surgery: a techial ischemia during noncardiac surgery: a technology assessment of transesophageal echocardiography and 12-lead

25 Shintani H, Nakano S, Matsuda H, Sakai K, Taniguchi K, Kawashima Y. Efficacy of transesophageal echocardiography as a perioperative monitor in patients undergoing cardiovascular surgery: analysis of 149 consecutive studies. $f$ Cardiovasc Surg 1990; 31: 564-70.

26 Iliceto S, Marangelli V, Memmola C, Rizzon P. Transesophageal Doppler echocardion evaluation of coronary blood flow velocity in baseline conditions and during velocity in baseline conditions and during dipyridamole83: $61-9$. 